\title{
¿Cómo generar calidad de gestión docente en las universidades públicas?
}

How to generate quality teaching management in public universities?

1 Arturo Francisco Ordoñez Peña Universidad Nacional de Tumbes, Perú aordonezp@untumbes.edu.pe

2 Jimy Humpiri Núñez https://orcid.org/0000-0002-4904-1781 Universidad Andina Néstor Cáceres Velásquez, Puno, Perú. jepistemologia@gmail.com

3 Raúl Andrés Villao Vera

Universidad Estatal Península de Santa Elena, Santa Elena, Ecuador rvillao@upse.edu.ec

Artículo de Investigación Científica y Tecnológica Enviado: 07/12/2021

Revisado: $22 / 12 / 2021$

Aceptado: 28/01/2022

Publicado:05/02/2022

DOI: https://doi.org/10.33262/ap.v4i1.1.154

\section{Cítese:}

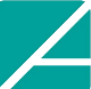

Ciencia Digital
Ordoñez Peña, A. F., Humpiri Núñez, J., \& Villao Vera, R. A. (2022). ¿Cómo generar calidad de gestión docente en las universidades públicas? . AlfaPublicaciones, 4(1.1), 188207. https://doi.org/10.33262/ap.v4i1.1.154

ALFA PUBLICACIONES, es una Revista Multidisciplinar, Trimestral, que se publicará en soporte electrónico tiene como misión contribuir a la formación de profesionales competentes con visión humanística y crítica que sean capaces de exponer sus resultados investigativos y científicos en la misma medida que se promueva mediante su intervención cambios positivos en la sociedad. https://alfapublicaciones.com

La revista es editada por la Editorial Ciencia Digital (Editorial de prestigio registrada en la Cámara Ecuatoriana de Libro con No de Afiliación 663) www.celibro.org.ec

Esta revista está protegida bajo una licencia Creative Commons Attribution Non Commercial No Derivatives 4.0 International. Copia de la licencia: http://creativecommons.org/licenses/by-nc-nd/4.0/ 
Palabras

claves:

educación, gestión, calidad, gestión docente

\section{Resumen}

El objetivo general es analizar la importancia de cómo generar una calidad de gestión docente que permita conseguir unos profesionales aptos para solucionar los problemas actuales con una conciencia propia y generadora de desarrollos sostenibles para el mundo. La metodología a emplear en esta investigación es de un diseño bibliográfico de tipo documental. La gestión educativa y la calidad educativa buscan desarrollar nuevas formas de generar estrategias que permitan fomentar habilidades y conocimientos, a través de la aplicación y guía de normas internacionales que ayuden a orientar el camino correcto para una educación productiva. Lo anterior no se cumple si la gestión docente no tiene el protagonismo necesario para tales cambios. La gestión docente debe planificar y controlar las actividades, estrategias y métodos que permiten una educación de calidad. Armonizar las estrategias con los cambios sostenibles necesarios para la sociedad y con el cumplimiento de las nuevas estrategias tecno pedagógicas son las bases para poder crear líderes que sean capaces de ofrecer climas acordes para cada tipo de ambiente. Todo lo anterior permite que la gestión docente tenga significado en los tiempos modernos, en el desarrollo de conocimientos y en la formación de profesionales acordes a las necesidades del sector productivo y de la sociedad.

\section{Keywords: \\ education, management, quality, teaching management.}

Abstract
The general objective is to analyze the importance of how to generate a quality of teaching management that allows for professionals capable of solving current problems with their own awareness and generating sustainable development for the world. The methodology to be used in this research is a documentary-type bibliographic design. Educational management and educational quality seek to develop new ways of generating strategies that foster skills and knowledge, through the application and guidance of international standards that help guide the right path for a productive education. The foregoing is not fulfilled if the teaching management does not have the necessary protagonist for such changes. Teaching management must plan and control the activities, strategies and methods that allow quality education. Harmonizing the strategies with the sustainable changes necessary for society and with the fulfillment of the new techno-pedagogical strategies are the bases to be able to create leaders who are capable 
of offering suitable climates for each type of environment. All of the above allows teaching management to have meaning in modern times, in the development of knowledge and in the training of professionals according to the needs of the productive sector and society.

\section{Introducción}

Existen aspectos a considerar dentro de cada proceso, procedimiento, técnica o método que se quiere aplicar. Muchas de estas generan una productividad que tendrá un fin que pudiera ser el incremento del rendimiento económico, financiero, académico, deportivo, entre otros. Para que suceda esta situación, es necesario la modificación de las variables que permitan alterar las condiciones de cada uno de los factores que inciden en dicha productividad. Por tal razón es necesario la aplicación de una gestión que permita ofrecer un desarrollo eficiente y eficaz de cada uno de estos parámetros.

En este sentido, es necesario poder desarrollar y analizar conceptualmente la importancia de la gestión en dichos procesos, procedimientos, técnicas o métodos. La palabra gestión proviene de "gestus", una palabra latina que significa: actitud, gesto, movimiento del cuerpo; asimismo, proviene directamente de "gestio-onis": acción de llevar a cabo y, además, está relacionada con "gesta", en tanto historia de lo realizado, y con "gestación", llevar encima.

Del mismo modo, la gestión abarca un amplio concepto para el desarrollo de las actividades de cualquier organización. La gestión es entendida como el sistema de actividades estratégicas y planificadas que se llevan a cabo guiadas por instrucciones y técnicas apropiadas para obtener determinados fines o metas (Vega-Gutiérrez, 2020). Igualmente, el concepto de gestión incluye las funciones de planificación, organización, evaluación y mejora, coordinadas con una fórmula de liderazgo o autoliderazgo, en todos los niveles y procesos de la organización (Cidad-Maestro, 2004).

De lo anterior, se puede definir que la gestión es uno de los baluartes que se deben considerar en cada organización con el fin de generar la calidad en cada una de las acciones que se realizan dentro de la misma. Desde este punto de vista, según Ramírez (2017, citado por Arias-González, 2018), se entiende por gestión la administración o dirección de una empresa, así como la combinación de conocimientos y competencias utilizando recursos económicos, tecnológicos y humanos con miras a alcanzar un objetivo determinando. 
Por lo cual, poder evaluar la gestión dentro de una organización requiere de métodos que permitan cuantificar sus rendimientos. Por tal motivo, los indicadores son los encargados de poder medir la gestión.

Todas las actividades pueden medirse con parámetros que enfocados a la toma de decisiones son señales para monitorear la gestión, así se asegura que las actividades vayan en el sentido correcto y permiten evaluar los resultados de una gestión frente a sus objetivos, metas y responsabilidades. (Pérez-Jaramillo, 2003, p.1)

Estos indicadores tienen ciertas dimensiones que abarcan el enfoque y el propósito, las cuales se pueden visualizar a través de la tabla 1.

Tabla 1

Dimensiones de los indicadores de gestión

\begin{tabular}{|c|c|c|c|}
\hline TIPO & REVISIÓN & ENFOQUE & PROPÓSITO \\
\hline $\begin{array}{l}\text { Planeación } \\
\text { Estratégica }\end{array}$ & $\begin{array}{l}\text { Desempeño global de } \\
\text { la organización. }\end{array}$ & Largo plazo & $\begin{array}{l}\text { Alcances de la visión y la } \\
\text { misión. }\end{array}$ \\
\hline $\begin{array}{l}\text { Planeación } \\
\text { Funcional }\end{array}$ & $\begin{array}{l}\text { Desempeño de las } \\
\text { áreas funcionales. }\end{array}$ & $\begin{array}{l}\text { Corto y mediano } \\
\text { plazo (Mensuales o } \\
\text { Semestrales) }\end{array}$ & $\begin{array}{l}\text { Apoyo de las áreas } \\
\text { funcionales para el logro de } \\
\text { las metas estratégicas de la } \\
\text { organización. }\end{array}$ \\
\hline $\begin{array}{l}\text { Planeación } \\
\text { Operativa }\end{array}$ & $\begin{array}{l}\text { Desempeño } \\
\text { individual de } \\
\text { empleados, equipos, } \\
\text { productos, servicios y } \\
\text { procesos. }\end{array}$ & $\begin{array}{l}\text { Cotidiano } \\
\text { (semanales, diarias, } \\
\text { horas) }\end{array}$ & $\begin{array}{l}\text { Alineamiento del desempeño } \\
\text { de empleados, equipos, } \\
\text { productos, servicios y de los } \\
\text { procesos con las metas de la } \\
\text { organización y de las áreas } \\
\text { funcionales. }\end{array}$ \\
\hline
\end{tabular}

Fuente: Pérez-Jaramillo (2003)

La aplicación de los indicadores de gestión y las respuestas que pueden ofrecer a la organización es de vital importancia, por lo que esto permite generar un control sobre las diversas situaciones que se pueden afrontar. Es aquí donde el control de gestión es parte clave en el desarrollo de las actividades. Según Dextre-Flores \& Del Pozo-Rivas (2012), el control es aquella función que pretende asegurar la consecución de los objetivos y planes prefijados en la fase de planificación.

Así mismo, gestionar implica una articulación de procesos y resultados, y también de corresponsabilidad y cogestión en la toma de decisiones, en contraposición al problema de la verticalidad / horizontal. El control permite gestionar los procesos, procedimientos, 
métodos y técnicas para generar la productividad y la calidad en la organización. De este particular, la calidad es un factor considerable a desarrollar en cualquier organización, porque es la búsqueda constante de la misma y de la cual se desprende los valores colaterales como el incremento de la productividad, la efectividad, la eficiencia y el rendimiento. Según Cidad-Maestro (2004) señala una característica importante de la calidad en la organización:

La función «calidad» ya no consistirá solamente en realizar la elemental función técnica de verificar el producto terminado o la más compleja de controlar el proceso de diseño y realización del producto, sino que se hará también responsable de que el resto de los procesos que no añaden directamente valor de cliente, incorporen el discurso y las prácticas de la calidad, porque lo añaden indirectamente, como ocurre con los procesos estratégicos — políticas, planificación, revisiones por la dirección, etc. - y los de apoyo —administración, formación, mantenimiento, etc.participando todos en su construcción. (p. 649)

La puesta a, punto de la calidad en cualquier organización requiere principalmente de compromisos por parte de las autoridades y de las personas involucradas en la misma. Es la necesidad de mejora constante para poder lograr los objetivos planteados. La puesta en marcha de la calidad requiere:

- La definición de una política explícita para la calidad.

- El reconocimiento del cliente como el factor clave que define los lineamientos de la calidad.

- El reconocimiento del factor humano como el eje del cambio y de la generación de valor.

- La innovación tecnológica como el sendero de expansión necesario.

- La cultura de la incorporación permanente de desafíos sustentada en la participación y en un ambiente laboral sano que promueva la creatividad (Malvicino, 2001, p.7).

Esta gestión y medición de la calidad abarca el sistema educativo, donde existen factores que buscan aumentar la productividad y el rendimiento, tanto de los sistemas, del recurso humano y de los estudiantes. El sistema educativo debe buscar siempre la eficiencia de sus procesos, tanto ejecutado por las políticas y mecanismos implementados por los directivos de los diversos planteles e instituciones como las estrategias pedagógicas planteadas por los docentes en las diferentes aulas de clase.

Estos lineamientos de la gestión educativa deben ir en función de la formación profesional de los estudiantes para que puedan solventar los problemas y necesidades de la sociedad y del sector industrial y productivo. En otras palabras, la gestión educativa se considera 
como un cuerpo organizado de principios y regularidades que se aplican para guiar y prescribir como han de funcionar y gestionar para cumplir con los propósitos y así conseguir la mayor eficacia y eficiencia posible (García-Liscano, 2014).

De la misma forma, la gestión educativa tiene dentro de sus aristas la gestión docente aplicada por los profesores de los diversos niveles educativos. Esta gestión docente debe buscar armonizar las estrategias pedagógicas de enseñanza, vincular las herramientas técnicas y aprovechar las virtudes de las Tecnologías de Información y Comunicación (TIC) con el objeto de generar los conocimientos en los estudiantes. Según la investigación de Salguero (2008), la gestión docente orientada hacia el éxito debe generar procesos de aprendizajes del tipo "hacer", mediante formas progresiva y constructivas de cambios de conducta interna o externa y dentro del contexto académico con acciones didácticas y pedagógicas.

Por lo cual, la gestión docente debe llevar un control y por ende generar una calidad en la educación que se desea obtener en los sistemas y en la sociedad, donde los estudiantes son fundamentales para su este desarrollo y para su propio beneficio. En tal sentido, recae la importancia de cómo generar una calidad de gestión docente que permita conseguir unos profesionales aptos para solucionar los problemas actuales con una conciencia propia y generadora de desarrollos sostenibles para el mundo. La metodología a emplear en esta investigación es de un diseño bibliográfico de tipo documental.

\section{Metodología}

La metodología aplicada en esta investigación es de carácter bibliográfico, debido a que se sustenta en las diversas investigaciones realizadas a través de documentos web, artículos científicos, trabajados de grado de diversas universidades, informes técnicos, guías entre otros. Según Hurtado (2010) el investigador lee, revisa las teorías y los estudios previos y analiza los planteamientos relacionados con su evento para interpretar los contenidos, juzga, valora y selecciona el material bibliográfico y las ideas relacionadas con su investigación. Es decir, la revisión de la literatura consiste en detectar, consultar y obtener la bibliografía y otros materiales útiles para los propósitos del estudio, de los cuales se extrae y recopila información relevante y necesaria para el problema de investigación (Hernández-Sampieri et al., 2014).

El procedimiento metodológico se basó en la consecución de los objetivos de la investigación los cuales destacan la importancia y características de la gestión, control de gestión, gestión de calidad, gestión educativa y gestión docente, así como el análisis a los parámetros más importantes de la gestión docente y cómo influye en la educación universitaria pública. 


\section{Resultados y Discusión}

\section{Características de la gestión educativa y calidad educativa}

Las instituciones educativas buscan generar políticas, mecanismos, estrategias, didácticas, métodos y técnicas que buscan mejorar la educación que se presenta en las sociedades. Obtener un rendimiento elevado de la educación en función de su productividad, eficiencia y eficacia es una de las claves y para ello se debe sincronizar una gestión de calidad de todo el sistema educativo. Es aquí donde la gestión educativa debe tomar la batuta y analizar cada uno de los parámetros que rigen en el sistema para que la educación sea de calidad y donde el principal recurso, tanto estudiantes y docentes, estén formándose en pro de una sociedad más sostenible acorde a los lineamientos exigidos en la actualidad.

Del mismo modo, el objetivo común de un sistema educativo de calidad es el aumento del rendimiento académico de los estudiantes. Para tal fin, se deben cumplir ciertas condiciones que permitan que dicho objetivo pueda cumplirse y el cual se vuelve un sistema complejo por las variables que se manejan. Estas son: alimentación, vivienda, materiales, biblioteca, vestimenta, doble jornada laboral, clima escolar positivo, docentes especializados, experiencia docente, gestión y liderazgo del director, acompañamiento técnico, programas de refuerzos; sin considerar el nivel socioeconómico (DIGEDUCA, 2018).

Para poder lograr que gran parte de estos factores se cumplan se requiere de una excelente gestión, tanto de los entes gubernamentales, de la empresa privada y de las direcciones educativas. En este sentido, es importante tener bien definido el concepto de lo que se refiere gestión, con el objetivo de poder aplicar bien dicho parámetro en el sistema educativo (Pareja, 2020). La gestión es el todo, ya que articula procesos teóricos y prácticos para favorecer el mejoramiento continuo de la calidad, equidad y pertinencia de la educación (García et al., 2018). De la misma forma, la Gestión, por consiguiente, implica un fuerte compromiso de sus actores con la institución y también con los valores y principios de eficacia y eficiencia de las acciones ejecutadas (García-Liscano, 2014).

Asimismo, la gestión educativa se entiende como los procesos de planificación, administración, seguimiento y evaluación, siempre entendidos como la base de una medición para la dirección escolar (García-Liscano, 2014). Del mismo modo, es un proceso orientado al fortalecimiento de los proyectos educativos de las instituciones, que ayuda a mantener la autonomía institucional, en el marco de las políticas públicas, y que enriquece los procesos pedagógicos con el fin de responder a las necesidades educativas locales (López-Paredes, 2017). Igualmente, esta gestión educativa tiene unos principios que están relacionado con la dirección de los planes educativos, las estrategias aplicadas por los docentes, los aprendizajes de los estudiantes, de los mecanismos aplicados por las 
instituciones para generar educación de calidad. Los principios que se pueden distinguir en la gestión educativa se desarrollan en la tabla 2.

Tabla 2

\section{Principios de la gestión educativa}

\begin{tabular}{|c|c|}
\hline PRINCIPIOS & CARACTERÍSTICAS \\
\hline $\begin{array}{c}\text { Gestión centrada en los } \\
\text { estudiantes }\end{array}$ & $\begin{array}{l}\text { La educación de los alumnos es la razón de ser, que el primer y último } \\
\text { objetivo de una institución escolar; por lo tanto: todas las acciones de } \\
\text { conducción o de dirección deben ser canalizadas para lograr este objetivo } \\
\text { institucional. }\end{array}$ \\
\hline $\begin{array}{l}\text { Jerarquía y autoridad } \\
\text { claramente definida }\end{array}$ & $\begin{array}{l}\text { Permite garantizar la unidad de acción de la organización, en la que la } \\
\text { dirección ejerce funciones, como tal: dirige, impulsa, ordena, sin disminuir } \\
\text { las competencias propias de cada instancia. }\end{array}$ \\
\hline $\begin{array}{l}\text { Claridad en la definición } \\
\text { de canales de } \\
\text { participación }\end{array}$ & $\begin{array}{l}\text { Para que la participación de los miembros de la comunidad educativa } \\
\text { guarde coherencia con los objetivos institucionales, se debe establecer el } \\
\text { sistema bien definido. Cada miembro debe conocer las formas. Los } \\
\text { momentos de su participación y la contribución coherente que éste debe } \\
\text { tener con los objetivos institucionales. Saber dónde, cuándo, cómo, por qué } \\
\text { participar y qué resultados pueden esperar. }\end{array}$ \\
\hline $\begin{array}{l}\text { Ubicación del personal de } \\
\text { acuerdo a su competencia } \\
\text { y/o especialización }\end{array}$ & $\begin{array}{l}\text { Se refiere a la necesidad de tomar en cuenta las habilidades y competencias } \\
\text { de cada persona, para considerar su ubicación en el lugar en que tendrá } \\
\text { mejor rendimiento y realización, que contribuirá a optimizar el } \\
\text { funcionamiento de la organización. }\end{array}$ \\
\hline $\begin{array}{l}\text { Coordinación fluida y } \\
\text { bien definida }\end{array}$ & $\begin{array}{l}\text { Establecer instancias de coordinación ágil y oportuna, mejora la } \\
\text { sincronización de acciones, evita esfuerzos innecesarios y permite una } \\
\text { mejor acción conjunta. }\end{array}$ \\
\hline $\begin{array}{c}\text { Transparencia y } \\
\text { comunicación permanente }\end{array}$ & $\begin{array}{l}\text { Todas las acciones que se realicen a nivel de centro educativo deben ser } \\
\text { conocidas por los miembros de la comunidad, de ahí la necesidad de contar } \\
\text { con mecanismos de comunicación. }\end{array}$ \\
\hline $\begin{array}{l}\text { Control y evaluación } \\
\text { eficaces y oportunos para } \\
\text { un mejoramiento continuo }\end{array}$ & $\begin{array}{l}\text { El control debe proporcionar información que oriente de manera oportuna } \\
\text { la decisión y asegure la dirección que tomen las tareas en función de los } \\
\text { objetivos }\end{array}$ \\
\hline
\end{tabular}

Fuente: Bedoya-Campos et al. (2021)

Por supuesto, que parte de estos principios deben ser liderizados por los directivos de las diversas instituciones públicas de educación superior. Estas políticas deben ir en función del desarrollo eficiente de la academia, a través del seguimiento y control de las funciones tecno pedagógicas utilizadas para el desarrollo del conocimiento. Según Contreras et al. (2016), la gestión de la directiva se fundamenta:

La gestión pedagógica del directivo debe centrarse en la mejora de los aprendizajes significativos a través de los docentes quienes orientan y dirigen la planificación escolar, considerando estrategias para el trabajo pedagógico a desarrollarse en la institución educativa; los cuales tendrán repercusión en la mejora de los resultados académicos y por ende en la calidad educativa. 
Ante esta situación, la gestión de la directiva de las instituciones universitarias debe fundamentarse en las dimensiones de gestión pedagógica enfocadas en las condiciones con que se desarrolla la educación y en los procesos pedagógicos desarrollados por los docentes, los cuales se detallan en la tabla 3 .

Tabla 3

Dimensiones de la gestión pedagógica de los directivos de las instituciones universitarias

\begin{tabular}{cl}
\hline DIMENSIONES & \multicolumn{1}{c}{ CARACTERÍSTICAS } \\
\hline \multirow{2}{*}{$\begin{array}{c}\text { Eximensten condiciones que se desarrollan desde una eficaz planificación hasta } \\
\text { las condiciones }\end{array}$} & $\begin{array}{l}\text { eldesarrollo de climas favorables con participación articulada de todos los } \\
\text { actores educativos a fin de que los estudiantes accedan a los procesos } \\
\text { educativos en condiciones de equidad, lo cual aún es un desafío latente del } \\
\text { sistema educativo los países del mundo. }\end{array}$ \\
\hline
\end{tabular}

Según el Ministerio de Educación del Perú (2013), comprende las competencias del directivo enfocadas hacia el desarrollo de la profesionalidad docente y el proceso de acompañamiento sistemático al docente para la mejora de los aprendizajes.

Fullan (1995) señaló que el asunto de re culturizar la escuela es desarrollar culturas de trabajo colaborativo que se focalicen de un modo sostenido en

Dimensión II: Gestión de los procesos pedagógicos la preparación continua y el desarrollo profesional de los profesores en relación con crear y valorar condiciones de aprendizaje para todos los estudiantes.

Los procesos pedagógicos se desarrollan frecuentemente de modo distinto a como fueron inicialmente planificados. Por ello, el cambio y la corrección deben ser posibles en cada momento, y debe existir suficiente libertad de actuación para repensar de nuevo las metas fijadas; debiendo existir espacios donde los docentes comparten sus prácticas más eficaces o reflexionan sobre aquellas que no lo son.

Fuente: Quispe (2018)

Dentro de esa gestión pedagógica se encuentra una serie de acciones que permiten generar una educación acorde a las necesidades que requiere la sociedad. Para lograr que dichas operaciones y realicen es necesario la aplicación de una planificación estratégica. El desarrollo de una planificación estratégica produce beneficios relacionados con la capacidad de realizar una gestión más eficiente, liberando recursos humanos y materiales, lo que redunda en la eficiencia productiva y en una mejor calidad de vida y trabajo para todos los miembros.

La planificación estratégica debe buscar solventar los inconvenientes y dificultades de la educación superior que pueden afectar el mismo proceso. Según Huerto Marimón (2012 citado por Batista de los Ríos, 2017), estos son: la insuficiente preparación y motivación de los recursos humanos, la inexistencia de una estrategia de comunicación social como soporte de la actividad, aspectos que inciden en el logro de resultados en la formación integral del estudiante y la transformación de los contextos. 
Para solventar estas debilidades es conveniente que la planeación estratégica tenga propuestas innovadoras que permitan el desarrollo de la educación con calidad. Estas innovaciones deben involucrar los desempeños de los directivos y docentes, así como de los ambientes educativos a los cuales deben estar los estudiantes. Según Bonilla (2010 citado por Chen-Quesada et al., 2020), las innovaciones son interactivas, sociales y surgen de las relaciones de los diferentes miembros de la sociedad y de esta con la naturaleza, por lo que se entiende que las innovaciones no surgen espontáneamente son la acumulación de un proceso de aprendizaje.

La aplicación de la innovación, sea pedagógica, en los procesos y procedimientos administrativos y en el desarrollo tecnológico, permitirá incrementar la calidad educativa en las instituciones de educación superior. La innovación trae consigo fortalezas en el marco de la gestión educativa, en especial la gestión docente; sin embargo, es necesario el compromiso y motivación ejercida por los docentes y de las autoridades educativas, lo cual se traduce en calidad educativa.

Por otra parte, es importante señalar las características ideales que se desean en la educación universitaria pública, según Vidal-Ledo \& Morales-Suárez (2010),

las cuales son:

- Ser accesible.

- Facilitar los recursos personales, organizativos y materiales requeridos.

- Promover cambio e innovación a través de la reflexión compartida sobre la propia práctica docente y el trabajo colaborativo del profesorado.

- Promover la participación activa del alumnado, tanto en el aprendizaje como en la vida de la institución, en un marco de valores donde todos se sientan respetados y valorados como personas.

- Lograr la participación de las familias e insertarse en la comunidad.

- Estimular y facilitar el desarrollo y el bienestar del profesorado, así como de los demás profesionales del centro. (pp. 253-254)

Lo anterior corresponde a las características de la calidad educativa. Igualmente, la calidad educativa la hacen las personas, tanto directivos, técnicos, administrativos, como docentes, quienes conociendo el uso y aplicación de las técnicas actuales de calidad para que puedan aplicarlas mejorando permanentemente su gestión (Senlle \& Gutiérrez, 2005). De la misma forma, la calidad de la educación no deja de ser un concepto normativo y multifactorial, donde intervienen dimensiones de la filosofía, la pedagogía, la cultura, la sociedad y la economía (Nicoletti, 2008).

Bajo estos múltiples factores, el concepto y aplicación de una calidad educativa es un sistema complejo que depende de muchas variables. Desde un punto ideal, el concepto de 
calidad como excelencia, basado en la definición tradicional, equivalente a poseer estudiantes sobresalientes, académicos destacados, y aseguramientos del primer nivel (Aguila, 2005). Lo que es muy ambiguo para la educación superior en Latinoamérica y el Caribe debido a los factores antes mencionados. Poder llegar a satisfacer cada necesidad requiere de un compromiso competo entre todas las aristas de la sociedad, lo cual lo hace difícil según sus vivencias y desarrollos.

En este particular, según Casassus (1999 citado por Quintana-Torres, 2018), como un concepto estratégico en las formulaciones de política educativa en la gran mayoría de los países, en torno al cual se estructura el resto de las políticas educativas. De lo anterior, se puede decir que las políticas deben considerar evaluaciones de los parámetros que influyen en el sistema educativo y por el cual los resultados son las respuestas a la situación presentada para cada sociedad y su educación. Esta visión solo ve la calidad educativa desde el punto de vista del rendimiento socioeconómico. Según Martin-Calvo (2018), esta definición genera una interpretación restringida a la medida, la cual no tiene en cuenta los sistemas actuales de baja calidad y el contexto social, aspectos que generan la importancia de plantear razonamientos no económicos respecto a la evaluación de la calidad educativa.

La aplicación de la calidad en la educación definitivamente debe llevarse en la evaluación de ciertos indicadores que manifiesten la realidad de la misma y así poder desarrollar las estrategias de control y mejora. González-Galán (2004 citado por Egido-Gálvez, 2005), señala que es poco congruente hablar de calidad sin hablar de evaluación, puesto que tildar una cosa como algo que tiene calidad exige realizar una medida, compararla con un referente ideal y elaborar un juicio sobre la adecuación del objeto o sujeto evaluado al referente utilizado

Uno de las guías metodológicas aplicadas para evaluar la calidad en cualquier organización es la ISO 9000. Esta misma técnica ha desarrollado mecanismos para implementarla en los sistemas educativos. La conclusión que arroja es que la calidad educativa presenta 3 dimensiones principales. Según Yzaguirre-Peralta (2005):

"La primera dimensión es la eficacia: una educación de calidad es aquella que logra que los estudiantes realmente aprendan lo que se supone deben aprender -aquello que está establecido en los planes y programas curriculares-, al cabo de determinados ciclos o niveles. Una segunda dimensión es la pertinencia en términos individuales y sociales, donde la educación de calidad es aquella cuyos contenidos responden adecuadamente a lo que el individuo necesita para desarrollarse como persona -intelectual, afectiva, moral y físicamente-, y para actuar en los diversos ámbitos de la sociedad -el político, el económico, el social-. La tercera dimensión es la que se refiere a los procesos y medios que el sistema brinda a los alumnos para 
el desarrollo de su experiencia educativa. Desde esta perspectiva una educación de calidad es aquella que ofrece al estudiante un adecuado contexto físico para el aprendizaje, un cuerpo docente convenientemente preparado para la tarea de enseñar, buenos materiales de estudio y de trabajo, estrategias didácticas adecuadas etc.” (p. 1)

Existe una cuarta dimensión, según la investigación realizada por Tünnermann-Bernheim (2008), que trata sobre la internacionalización de la educación. Este autor manifiesta que esta dimensión es la que hace indispensable la existencia de sistemas de acreditación, a fin de establecer los mecanismos adecuados para el aseguramiento de la calidad no solo de los ofrecimientos de educación superior a nivel nacional, sino también a nivel internacional. Esta dimensión toma como medidor de la calidad el sistema de acreditación, la cual se basa en normas aprobadas internacionalmente y en las cuales las universidades públicas deben cumplir para poder generar una educación de calidad.

Por lo cual, la acreditación como mecanismo de aseguramiento de la calidad, en la medida en que sea asumida por las instituciones como parte de su cultura, puede ser un dispositivo apto para el mejoramiento de la calidad de la educación superior (Orozco-Silva, 2010). Asimismo, la acreditación es uno de los instrumentos que pudieran medir la calidad de la educación superior, por lo que su evaluación o medición es parte importante para cumplir con los estándares arrojados por las entidades normativas internacionales y por los entes gubernamentales correspondientes.

En este sentido, la acreditación para la búsqueda de la calidad educativa en las universidades públicas debe ser el horizonte de la gestión educativa aplicada por las instituciones públicas correspondientes, por los directivos de las mismas y los docentes como facilitares de la información. Según Dias-Sobrinho (2009):

La calidad de la educación superior está directamente relacionada con su capacidad de contribuir al desarrollo de los individuos y de las sociedades. La formación integral de los individuos se correlaciona con el desarrollo humano social, el cual requiere un amplio incremento de la escolaridad de la población, en términos de cobertura y calidad, una fuerte revivificación de las políticas de aumento de la equidad y de disminución de la pobreza, y estrategias de aprovechamiento de los recursos naturales y de aplicación de los conocimientos para el desenvolvimiento sostenible. Esto requiere recursos materiales y humanos, es decir, financiamiento, voluntad política y capacidades intelectuales y éticas de los Estados, de la sociedad y de las instituciones. (p.98) 


\section{Parámetros de la gestión docente}

Dentro de las características u objetivos de la gestión educativa para generar la calidad que se pretende en la educación es necesario planificar, desarrollar, evaluar y control la gestión docente en las universidades públicas. Este tipo de gestión es la última escala y la más importante para desarrollar la calidad de la educación. La gestión educativa es un término compuesto que integra la docencia, la investigación, la extensión y la gestión administrativa que acompaña sus tareas y procesos laborales en pro de unos objetivos e indicadores que atienden a un plan racional y medible en productos y procesos (Salguero, 2008). Según Morales-Zambrano (2020):

La gestión docente detalla la manera de manejar problemas y seguir trabajando hacia delante con la propuesta planteada cuando sea posible o buscar otras alternativas cuando no lo sea, no se enfoca en negar la realidad si no en trabajarla, defenderla y sacarla adelante con los recursos que se le presenten reconociendo sus dotes de buen gestor en la enseñanza de los estudiantes. (p.14)

Asimismo, el desarrollo de la gestión docente no es una tarea fácil, por lo que requiere del compromiso y motivación de los profesores. En este sentido, deben desarrollar ciertas destrezas y habilidades para poder gestionar sus funciones. Según Carmona (2003 citado por Cartagena \& Atencio, 2015), el proceso de gestión requiere de esfuerzos que deben estar orientados a reducir la improvisación, integrando comportamientos de actuación más organizativos y disciplinarios.

Una de las etapas de la gestión docente es la planificación de las actividades o acciones a realizar dentro y fuera del aula de clase en pro del desarrollo del conocimiento. En otras palabras, la planificación trata de un conjunto de cosas por ejecutar en un intervalo de tiempo y uso de recursos que seleccionará de la organización universitaria; así como la organización de los contenidos curriculares y disciplinares, las experiencias en el ejercicio, la consulta a diferentes fuentes de información, entre otros (Salguero, 2008).

Otro de los aspectos a considerar es el control de las mis actividades, estrategias y métodos pedagógicos implementados. El control es la base para que se pueda establecer técnicas acordes para el desarrollo del conocimiento. Por ejemplo, el mejorar el control ayudará a optimizar el tiempo que los docentes permitiendo participar de manera activa en proyectos de investigación, elaboración de artículos científicos, vinculación con la sociedad, tutorías pedagógicas, académicas, practicas preprofesionales, y de trabajos de titulación (López-Franco, 2019).

Del mismo modo, para lograr la eficiencia y eficacia de la gestión docente se requiere una constante preparación del cuerpo docente en sus áreas de conocimiento, lo que permitirá 
un desarrollo pedagógico de calidad. Parte de este objetivo tiene responsabilidad los entes gubernamentales y las instituciones de educación superior. Un posible aporte es la creación de oficinas de difusión que reglamenten y lleven adelante el recuento de las actividades académicas, estableciendo el seguimiento y la constante actualización, conformando un centro de excelencia académica y de aprendizaje que serviría para la promoción y divulgación del conocimiento (Ganuza et al., 2017).

Dicha preparación también debe ir en función de los objetivos propuestos a nivel mundial para garantizar la vida y los recursos a las futuras generaciones, por lo que deben estar orientadas a la sustentabilidad del medio ambiente. el conocimiento debe ir en función del cumplimiento de los 17 Objetivos del Desarrollo Sostenible (ODS) de la Organización de las Naciones Unidas (ONU). Parte de la idea de que la ambientalización universitaria pasa por ofrecer oportunidades para que los estudiantes incorporen en sus estilos de vida y en sus códigos deontológicos profesionales principios y fundamentos inspirados en las buenas prácticas proambientales (Gutiérrez \& González, 2005).

De la misma forma, el docente debe estar actualizado con metodologías innovadoras tanto en el desarrollo de estrategias pedagógicas como en el dominio de las herramientas tecnológicas impulsadas por las TIC. La utilización de las TIC en el desarrollo de la educación es fundamental, por lo cual se deben distinguir las ventajas y desventajas de su utilización, tal como se muestran en la Tabla 4. Este último es una parte fundamental en el desarrollo de los conocimientos en la actualidad, y más después de las consecuencias arrojadas por la pandemia por COVID-19, que precipitaron acciones de confinamiento y distanciamiento social. La educación virtual ha tomado gran protagonismo. Es por ello la necesidad de que los docentes dominen los métodos tecno pedagógicos y sean una de las prioridades en la planificación de su gestión. Además, la modernización educativa se planteó como una necesidad imperiosa de adecuar la formación y capacitación de los recursos humanos a las exigencias de las dinámicas aceleradas de los sistemas tecnoproductivos de las sociedades (Jiménez Ortiz, 2011).

\section{Tabla 4}

Ventajas y desventajas por el uso de las TIC en la educación superior

\begin{tabular}{ll}
\hline \multicolumn{1}{c}{ VENTAJAS } & \multicolumn{1}{c}{ DESVENTAJAS } \\
\hline Interacción sin barreras geográficas. & Distracciones \\
Diversidad de información. & Aprendizaje superficial. \\
Aprendizaje a ritmo propio. & Proceso educativo poco humano. \\
Desarrollo de habilidades. & No es completamente inclusivo \\
Fortalecimiento de iniciativa. & Puede anular habilidades y capacidad crítica. \\
Corrección inmediata & \\
\hline
\end{tabular}


Por consiguiente, todo lo anterior permitiría fomentar un liderazgo transformacional que seria responsabilidad del docente, haciendo o transformando a los estudiantes en lideres potencias dentro del aparato productivo y de la sociedad con la capacidad de solventar las inquietudes y resolviendo las necesidades más básicas de su entorno. Según Pedraja et al. (2012 citado por Contreras et al., 2016), señalan que el liderazgo transformacional conduce al logro de estándares de excelencia e impacta en el proceso de gestión de recursos humanos, el cual tiene relación directa con la calidad de los procesos de enseñanza y con el aprendizaje de los estudiantes.

En definitiva, la gestión docente que involucre los anteriores parámetros creará un ambiente favorable para la generación de conocimiento, por lo cual el clima organizacional se incrementa si se cumple con una eficiente gestión docente. El clima organizacional influye en las actividades, el comportamiento de sus miembros, y en el ambiente psicosocial en el que se desenvuelven los trabajadores de una organización determinada (Segredo, 2011).

\section{Conclusiones}

- Para lograr una gestión docente que pueda cumplir con los objetivos de las instituciones de educación superior debe primero poder generar una gestión eficiente, eficaz y productiva, en la cual se base en la planificación, aplicación, evaluación y control de sus funciones dentro y fuera del aula con el objeto de generar los conocimientos necesarios e importantes para el desarrollo de los profesionales que solventaran las necesidades de la sociedad. Cada uno de estos pasos requieres que se realicen a través de actividades de calidad, por lo que estas dos situaciones, gestión y calidad, permitirán obtener un sistema educativo acorde a los nuevos tiempos.

- La gestión educativa y la calidad educativa buscan desarrollar nuevas formas de generar estrategias que permitan fomentar habilidades y conocimientos, a través de la aplicación y guía de normas internacionales que ayuden a orientar el camino correcto para una educación productiva. Lo anterior no se cumple si la gestión docente no tiene el protagonismo necesario para tales cambios. La gestión docente debe planificar y controlar las actividades, estrategias y métodos que permiten una educación de calidad. Armonizar las estrategias con los cambios sostenibles necesarios para la sociedad y con el cumplimiento de las nuevas estrategias tecno pedagógicas son las bases para poder crear lideres que sean capaces de ofrecer climas acordes para cada tipo de ambiente. Todo lo anterior permite que la gestión docente tenga significado en los tiempos modernos, en el desarrollo de conocimientos y en la formación de profesionales acordes a las necesidades del sector productivo y de la sociedad. 


\section{Referencias Bibliográficas}

Aguila Cabrera, V. (2005). el concepto calidad en la educacion universitaria: clave para el logro de la competitividad institucional. Revista Iberoamericana de Educación, $1-7$.

Arias-González, I. P. (2018). Auditoría un enfoque de gestión. Observatorio de la Economía Latinoamericana.

Batista de los Ríos, D. (2017). Gestión pedagógica de la extensión universitaria para la formación integral del estudiante. Las Tunas: Tesis para optar al grado de Doctor en Ciencias Pedagógicas de la Universidad de Las Tunas.

Bedoya-Campos, Y. Y., Salinas-Loarte, E. A., Palomino-Torres, E. F., \& Sánchez-Solis, Y. (2021). Gestión pedagógica y calidad educativa en una universidad pública del perú.

Cartagena, C., \& Atencio, E. A. (2015). Destrezas de gestión docente para la planificación de proyectos educativos en la corporación tecnológica industrial de Colombia. REDHECS: Revista electrónica de Humanidades, Educación y Comunicación Social, 10(20), 59-72.

Chen-Quesada, E., Cerdas-Montano, V., \& Rosabal-Vitoria, S. (2020). Modelos de gestión pedagógica: Factores de participación, cambio e innovación en centros educativos costarricenses. Revista Electrónica Educare, 24(2), 317-345.

Cidad-Maestro, E. (2004). La gestión de la calidad en las organizaciones de educación superior. Aportación del enfoque de la Organización Internacional de Normalización (ISO). Revista Complutense de Educación. Vol. 15 Núm. 2, 647686.

Contreras, F. G., Villegas, F. V., Rejas, L. P., \& Ponce, E. R. (2016). Liderazgo transformacional y su incidencia en la gestión docente: el caso de un colegio en el norte de Chile. Interciencia, 41(9), 596-604.

Dextre-Flores, J. C., \& Del Pozo-Rivas, R. S. (2012). ¿Control de gestión o gestión de control? Contabilidad y negocios, 7(14), 69-80.

Dias-Sobrinho, J. (2009). Capítulo 3: calidad, pertinencia y responsabilidad social de la universidad latinoamericana y caribeña. Tendencias de la Educación Superior en América Latina y el Caribe. 
DIGEDUCA. (2018). Líneas de investigación educativas. Dirección General de Evaluación e Investigación Educativa (Digeduca), Ministerio de Educación de Guatemala.

Egido-Gálvez, I. (2005). Reflexiones en torno a la evaluación de la calidad educativa. Tendencias Pedagógicas 10, 17-28.

Ganuza, C. V., Rodríguez-Jara, R. E., Auccahuallpa-Fernández, R., Maldonado, L., Ávila-Larriva, M. P., \& García-Macías, E. (2017). Multidimensionalidad de la calidad en la educación superior del Ecuador: Parámetros para su análisis. Universidad Nacional de Educación del Ecuador.

García Colina, F. J., Juárez Hernández, S. C., \& Salgado García, L. (2018). Gestión escolar y calidad educativa. Revista Cubana de Educación Superior, 37(2), 206216.

García-Liscano, R. (2014). Estrategias de gestión educativa desde la perspectiva de la práctica de liderazgo y el fortalecer del buen vivir en la escuela de educación básica "narciso cerdo maldonado", del cantón la maná, provincia de cotopaxi. Quevedo, Ecuador: Trabajo de grado para optar al título de Magister en Docencia y Currículo de la Universidad Técnica de Babahoyo.

Gutiérrez, J., \& González, A. (2005). Ambientalizar la universidad: un reto institucional para el aseguramiento de la calidad en los ámbitos curriculares y de la gestión. Revista Iberoamericana de Educación, 1-14.

Hernández-Sampieri, R., Fernádez-Collado, C., \& Batista, M. d. (2014). Metodología d ela investigación, 6ta edición. México, D. F.: mcgraw-hill / interamericana editores, s.a. de c.v.

Hurtado, J. (2010). Metodología de la investigación; Guía para una comprensión holística de la ciencia, 4ta edición. Caracas, Venezuela: Quirón Ediciones.

Jiménez Ortiz, M. D. (2011). El discurso mundial de modernización educativa: evaluación de la calidad y reforma de las universidades latinoamericanas. Espacio Abierto, 20(2), 219-238.

López-Franco, N. Y. (2019). Estrategias de control para mejorar la gestión docente en la Universidad de Guayaquil-Guayaquil, 2018. Piura, Perú: Trabajo de grado para optar el título de Maestra en Administración de Negocios de la Universidad César Vallejo.

López-Paredes, M. A. (2017). La Gestión pedagógica. Apuntes para un estudio necesario. Dominio de las Ciencias, 3(1), 201-215. 
Malvicino, G. A. (2001). La gestion de la calidad en el ambito de la administracion publica. VI Congreso Internacional del CLAD sobre la Reforma del Estado y de la Administración Pública, (págs. 1-15). Buenos Aires - Argentina.

Martin-Calvo, J. F. (2018). Calidad educativa en la educación superior colombiana: una aproximación teórica. Sophia, 14(2), 4-14.

Morales-Zambrano, S. C. (2020). Gerencia educativa y gestión docente en una Institución Educativa de Guayaquil, 2020. Piura, Perú: Trabajo de grado para optar al título de Maestra en Administración de la Educación de la Universidad Cesar Vallejo.

Nicoletti, J. (2008). Adecuación y aplicación de las normas de calidad iso 9000:2000 en el campo educativo. Horizontes Educacionales, vol. 13, núm. 2, 75-86.

Orozco-Silva, L. E. (2010). Calidad académica y relevancia social de la educación superior en América Latina. RIES, Revista Iberoamericana de Educación Superior, 1(1), 24-36.

Pareja, M. Q. (2020). La gestión pedagógica en la mejora del desempeño docente. Investigación valdizana, 14(1), 7-14.

Pérez-Jaramillo, C. M. (2003). Los indicadores de gestión. España.

Quintana-Torres, Y. E. (2018). Calidad educativa y gestión escolar: una relación dinámica. Educación y educadores, 21(2), 259-281.

Quispe Ramos, R. Y. (2018). Gestión pedagógica y calidad educativa en instituciones educativas del nivel inicial de la Red 10-UGEL 01, 2018. San Juan de Lurigancho, Perú: Trabajo de grado para optar al título de Mestra en Educación mención Docencia y Gestión Educativa de la Universidad Cesar Vallejo.

Salguero, L. A. (2008). Gestión docente y generación de espacios organizacionales en las universidades. Laurus, 14(27), 11-32.

Segredo Pérez, A. M. (2011). La gestión universitaria y el clima organizacional. Educación médica superior, 25(2), 164-177.

Senlle, A., \& Gutiérrez, N. (2005). Calidad en los servicios educativos. Madrid - España: Ediciones Díaz de Santos.

Tünnermann-Bernheim, C. (2008). La calidad de la educación superior y su acreditación: la experiencia centroamericana. Avaliação, Campinas; Sorocaba, SP, 13(2), 313-336. 
Vega-Gutiérrez, L. V. (2020). Gestión educativa y su relación con el desempeño docente. Ciencia y Educación-Revista Científica, 1(2), 18-28.

Vidal-Ledo, M., \& Morales-Suárez, I. (2010). Calidad educativa. Educación Médica Superior, 24(2), 253-274.

Yzaguirre-Peralta, L. E. (2005). calidad educativa e iso 9001-2000 en méxico. REICE Revista Electrónica Iberoamericana sobre Calidad, Eficacia y Cambio en Educación. Vol. 3, No. 1, 421-431.

Zurita-Sibambe, A. D. (2018). El uso de las TIC en la gestión docente. Guayaquil, Ecuador: Trabajo de grado para optar al título de Licenciado en Ciencias de la Educación de la Universidad de Guayaquil.

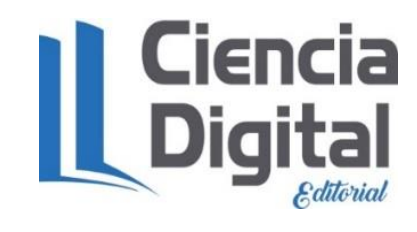


El artículo que se publica es de exclusiva responsabilidad de los autores y no necesariamente reflejan el pensamiento de la Revista Alfa Publicaciones.

\section{Ciencia}

El artículo queda en propiedad de la revista y, por tanto, su publicación parcial y/o total en otro medio tiene que ser autorizado por el director de la Revista Alfa Publicaciones.
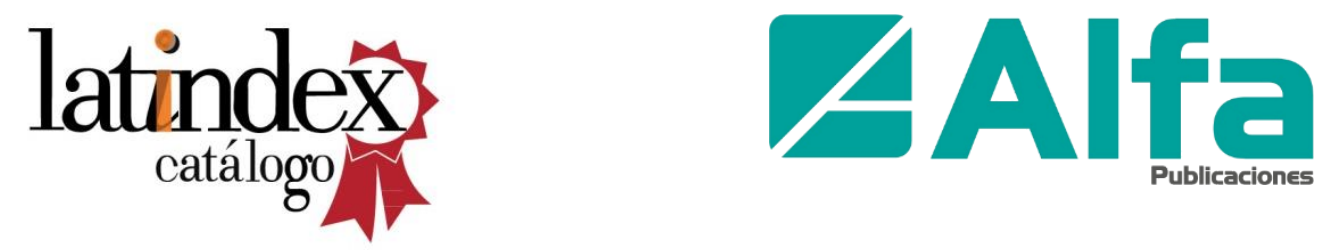

doi crosiret latindex TlatinREV
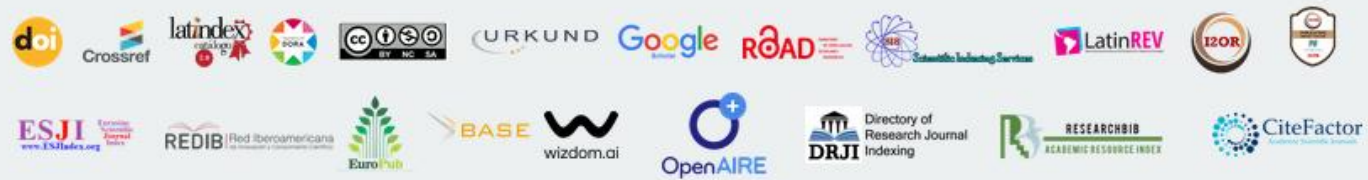